\title{
Outsourcing in der Lehre - Eine Möglichkeit für die Zukunft? ${ }^{1}$
}

\section{Outsourcing of Academic Teaching - A Perspective for the Future?}

\author{
Autor \\ C. C. Geilen \\ Institut \\ CCG Berlin GmbH
}

\section{Bibliografie}

Dol 10.1055/s-0028-1119641

Akt Dermatol 2009; 35:

151-156 @ Georg Thieme

Verlag KG Stuttgart · New York

ISSN 0340-2541

Korrespondenzadresse

Prof. Dr. med. Dr. rer. nat.

Christoph C. Geilen

CCG Berlin GmbH

Kurfürstendamm 21

10719 Berlin

christoph.geilen@charite.de

www.ccg-berlin.de

\section{Zusammenfassung}

Die steigende Lehrbelastung an deutschen Hochschulen verlangt in Anbetracht steigender Studentenzahlen nach innovativen Lösungsansätzen. Neben der Differenzierung in Forschungsund Lehrprofessuren, wie sie vom Wissenschaftsrat empfohlen wird, gibt es eine Reihe weiterer Möglichkeiten, den Anforderungen gerecht zu werden. Das anteilige Outsourcing des Lehrdeputats an externe Dozenten durch einen

\section{Einführung}

$\nabla$

In neuerer Zeit hat die Lehrbelastung an den deutschen Hochschulen erheblich zugenommen. Im Wintersemester 2008/2009 waren laut Statistischem Bundesamt erstmals seit 5 Jahren wieder über 2 Millionen Studierende an den Hochschulen in Deutschland immatrikuliert und die Erstsemesterquote erreichte mit rund 385500 Studierenden einen neuen Höchststand [1]. Das sind 39,3\% dieses Geburtenjahrgangs. Die Lehrbelastung für die Hochschullehrer stellt in Anbetracht dieser Zahlen unbestreitbar eine Herausforderung dar.

Forschung, Lehre, Prüfungen, Selbstverwaltung und in den Universitätskliniken die Krankenversorgung stellen ein System dar, dessen Koordinaten im Rahmen zunehmender Ökonomisierung und Bürokratisierung immer höhere zeitliche Anforderungen an die Hochschullehrerschaft stellen. Insofern wird es für Hochschulen zunehmend schwerer, gewünschte und teilweise auch notwendige neue Studiengänge einzuführen, Bachelor- und Masterstudiengänge umzusetzen oder Studiengänge zu reformieren, zumal das spezialisierten Dienstleister hat sich hierbei als erfolgreich erwiesen. Durch diese Professionalisierung der Lehre wird ein hoher Grad an Einheit von Forschung und Lehre erreicht und die geschaffenen Forschungsfreiräume könnten darüber hinaus dazu führen, dass forschungsaktive Hochschullehrer nicht an reine Forschungsinstitutionen abwandern und weiterhin mit ihrer Expertise zu einer hohen Qualität der universitären Lehre beitragen.

Lehrpersonal wegen Sparauflagen vielerorts eingeschränkt wird. Personelle Engpässe, beispielsweise im Zuge von Neu- oder Wiederberufungen, sind immer schwieriger für die verbliebenen Hochschullehrer abzudecken. In Deutschland werden immerhin nur ca. 30 Prozent der Berufungsverfahren innerhalb eines Jahres abgeschlossen [2], sodass Personallücken über einen längeren Zeitraum vertreten werden müssen.

Sicherlich lassen sich einzelne Vorlesungen oder Seminare durch kurzfristige Lehraufträge abdecken. Anders sieht es allerdings aus, wenn zentrale Lehreinheiten, wie Hauptvorlesungen, Pflichtseminare und Praktika konzipiert, koordiniert und durchgeführt werden müssen. Dies benötigt eine zentrale Koordination durch eine Fachvertretung. Im Normalfall erfolgt sie durch den oder die Lehrstuhlinhaber(in). Welche Möglichkeiten gibt es, wenn dies nicht erfolgen kann? Wie kann qualitativ hochwertige Lehre sichergestellt werden? An diesem Punkt ist die Frage zu klären, ob diese zentrale Position für eine begrenzte Zeit von externem Lehrpersonal oder einem geeigneten, erfahrenen Dienstleister übernommen werden kann.

\footnotetext{
${ }^{1}$ Diese Arbeit ist der „Berliner Stiftung für Dermatologie“ (BSD) gewidmet, die in diesem Jahr ihr 10-jähriges Jubiläum feiert.
} 


\section{Outsourcing von universitärer Lehre? $\nabla$}

Humboldts Idee der Einheit von Forschung und Lehre hat ohne Zweifel lange Zeit zur hohen Qualität von Universitäten geführt. Sie wird aber zunehmend skeptischer diskutiert, da mittlerweile die heutigen Anforderungen an gute Forschung auf der einen und gute Lehre auf der anderen Seite so weit auseinander laufen, dass eine Einheit, die beiden Seiten gleichermaßen gerecht wird, sehr schwer zu realisieren ist.

Ein wichtiger Aspekt dieses Auseinanderdriftens ist die zunehmende Zahl der Studierenden und der Umfang der Lehrinhalte. Während in den 50er-Jahren ca. 5\% einer Alterskohorte die Hochschulen besuchte, überstieg in den 90er-Jahren in vielen europäischen Ländern diese Rate die 20\%-Marke. In Deutschland, Frankreich, Italien und den skandinavischen Ländern wurde diese Marke noch überschritten [3]. Diese Entwicklung führte konsequenterweise zu einer steigenden Lehrbelastung, wodurch die Forschungskapazitäten der Hochschullehrerschaft in vielen Fällen eingeschränkt wurden.

Ungeachtet dieser Entwicklungen besteht auch heute noch in Deutschland der Anspruch auf eine Einheit von Forschung und Lehre. Dies soll aber nicht ein Grund sein, zeitlich begrenztes Outsourcing von Lehre von vornherein auszuschließen, wenn ein gewisser zeitlicher oder personeller Engpass überbrückt werden muss. Es stellt nur besondere Anforderungen an den externen Dienstleister. Dieser muss einen externen Dozentenpool unterhalten, der auch Vertretungen in Krankheitsfällen abdeckt, und sicherstellen, dass das Lehrpersonal neben einer qualifizierten, soliden wissenschaftlichen Basis auch über ausreichende Erfahrungen in der universitären Lehre verfügt. Ein Mangel geeigneter Fachkräfte für die universitäre Lehre besteht im Allgemeinen nicht, da aufgrund der zeitlichen Befristung der Beschäftigungsverhältnisse von wissenschaftlichen Mitarbeitern und Hochschulassistenten in Deutschland ein außerordentlich großes Reservoir an gut ausgebildetem Fachpersonal außerhalb und innerhalb von akademischen Einrichtungen besteht, das über genügend Zeit und teilweise auch über die Lehrbefugnis verfügt und dessen Potenzial für die Hochschullehre ungenutzt bleibt.

\section{Das Mannheimer Reformierte Curriculum Medizin (MaReCuM) \\ $\nabla$}

Diese Überlegungen sollen im Folgenden anhand eines Fallbeispiels konkretisiert werden. Zum Wintersemester 2006/2007 ging der humanmedizinische Reformstudiengang MaReCuM der Medizinischen Fakultät Mannheim der Universität Heidelberg erstmals mit 180 Studierenden im Erstsemester an den Start. Die Mannheimer Fakultät hatte bis dahin nicht das Grundstudium bis zur M1-Prüfung („Physikum“) angeboten, sondern von der Medizinischen Fakultät Heidelberg Studierende, nach deren bestandener M1-Prüfung, in die klinische Ausbildung übernommen.

Der Reformstudiengang MaReCuM gliedert sich in ein integriertes Grundstudium, in dem die Ausbildungsinhalte naturwissenschaftlich orientiert und organbezogen gelehrt werden und gleichzeitig naturwissenschaftliche Kenntnisse mit relevanten Inhalten des klinischen Studienabschnittes verknüpft werden. Der folgende klinische Studienabschnitt bietet neigungsorientierte Qualifizierungswege (Praxisorientierte Medizin, Humanmedizin mit zusätzlichem Masterabschluss und wissenschaftliches Arbeiten) an und schließt mit dem Praktischen Jahr, das sich in vier Quartale gliedert, ab [4].
Mit der Entscheidung einen Reformstudiengang ins Leben zu rufen, war der Übergang der 1964 gegründeten Mannheimer Fakultät in eine Vollfakultät vorgegeben. Insofern mussten sämtliche vorklinischen Fächer an der Mannheimer Fakultät personell neu besetzt werden. Hierbei konnte nur bedingt auf vorhandenes Lehrpersonal zurückgegriffen werden. Im Laufe der Vorbereitungs- und Berufungsphase kam es zu kurzfristigen Absagen von vorgesehenen Fachvertretern in den Fächern Zellbiologie/ Biologie sowie Biochemie. Die Mannheimer Medizinische Fakultät beschritt daraufhin einen innovativen Weg: Sie übertrug die Lehrverantwortung zeitlich befristet einem externen Dienstleister, der sich auf die Beratung und Durchführung im Bereich der medizinisch-naturwissenschaftlichen Lehre spezialisiert hatte. Es galt, die Lehrinhalte und Lernziele zu definieren, gemeinsam mit den anderen Fachvertretern der Vorklinik die Adaptation an die Vorgaben von MaReCuM vorzunehmen und die Stundenpläne zu entwickeln. Schließlich mussten für alle Vorlesungen, Seminare und Praktika der Fächer Biochemie, Zellbiologie und Biologie Lehrkonzepte und -materialien erstellt und qualifizierte Lehrkräfte bzw. Dozenten zur Verfügung gestellt werden. Ein wichtiger Punkt war hierbei, eine homogene Lehre trotz heterogener Dozentenschaft bei teilweise 8 parallelen Seminargruppen zu gewährleisten, da neben den Mitarbeitern des Dienstleisters, Mannheimer Dozenten und Dozenten anderer Herkunft integriert werden mussten. Insgesamt kamen 40 verschiedene Dozenten zum Einsatz. Um dies zu gewährleisten, wurde allen Seminardozenten ein fester Satz von Lehrfolien vorgegeben, die ungefähr 50\% der Unterrichtszeit einnahmen; die restliche Zeit konnte individuell von den Dozenten gestaltet werden. Gruppenarbeitsthemen und Referate ergänzten die Seminararbeit. Für das Fach Biochemie wurden in diesem Rahmen für 41 verschiedene Seminarthemen insgesamt 1230 Folien erstellt (๑ Tab. 1).

Das Fach Zellbiologie wurde nicht durch den externen Dienstleister vertreten, wie es in der Biochemie der Fall war, sondern durch den Fachvertreter der Anatomie, sodass nur die molekulare Zellbiologie und die Biologie extern sichergestellt werden musste. Immerhin wurden bei diesem Teilauftrag insgesamt 12 Vorlesungs- und Seminarthemen vermittelt und 360 Seminarfolien erstellt ( $\bullet$ Tab. 2).

Die Entwicklung und Durchführung des Praktikums Biochemie stellte eine besondere Herausforderung dar, da zwar moderne Lehrlaboratorien vorhanden waren, aber ein großer Teil der Geräte und Ausstattungen beschafft werden mussten. Zudem mussten die Praktikumsskripten entworfen und verfasst werden, sowie die Versuche den vorhandenen Labor- und Gerätebedingungen angepasst werden. Insofern wurden alle Praktikumsversuche mit den entsprechenden Geräten im Vorfeld durchgeführt und adaptiert. Schließlich wurden so 15 verschiedene biochemische Praktika mit 45 Versuchen entwickelt sowie 512 Praktikumsskript- und Protokollseiten verfasst ( Tab.3).

Insgesamt wurden pro Jahrgang im Fach Biochemie 86 Vorlesungsstunden und im Fach Zell- und Molekularbiologie 34 Vorlesungsstunden gehalten. Bei den Seminaren mit jeweils acht Seminargruppen wurden 544 bzw. 272 Stunden unterrichtet, auf Praxiseinheiten entfielen 60 bzw. 72 Unterrichtsstunden und auf die Praktika mit 12 Gruppen 576 bzw. 144 Stunden. Demnach mussten pro Jahrgang für 1786 Unterrichtsstunden Dozenten gefunden werden. Von Dozenten der Mannheimer Fakultät konnten im Fach Biochemie 316 von 1266 Stunden übernommen werden. Dies entspricht ungefähr $25 \%$. Im Fach Zellund Molekularbiologie war der Anteil mit 61\% (318/520 Stunden) 
Tab. 1 Seminarthemen im Fach Biochemie.

1. Molekulare Bausteine der Zelle

2. Rechnen in der Chemie und Biochemie

3. Nukleinsäuren

4. Aminosäuren und Proteine

5. Enzyme und Enzymkinetik

6. Kohlenhydrate

7. Mitochondrien und Atmungskette

8. Lipide

9. Prinzipien der Signaltransduktion

10. Extrazelluläre Matrix

11. Energiegewinnung und Muskelarbeit

12. Biochemie des Nervengewebes und der Muskelkontraktion

13. Calcium und Phosphatstoffwechsel

14. Blutgerinnung

15. Hämsynthese, Porphyrine, Eisenstoffwechsel, Antioxidantien

16. Stoffwechsel der Energiesubstrate

17. Hormonelle Steuerung der Herzfunktion

18. Lipoprotein-Stoffwechsel

19. Immunologie I

20. Immunologie II

21. Immunologie III

22. Immunologie IV

23. Biochemie der Verdauung

24. Gallensäuren und Biotransformation

25. Aminosäurenstoffwechsel

26. Gentechnik

27. Gendiagnostik

28. Intrazelluläre Signaltransduktionsmechanismen

29. Nukleotidstoffwechsel

30. Regulation der Genexpression

31. Mutation und Kanzerogenese

32. Methoden der Molekularmedizin

33. Einführung Hormone

34. Regulation des Intermediärstoffwechsels

35. Regulation der Hormonausschüttung

36. Mediatoren

37. Schilddrüse und lodstoffwechsel

38. Steroidhormone

39. Vitamine

40. Transmitter

41. Biochemie der Sinneswahrnehmungen

sehr viel höher. Insgesamt zeigte sich, dass die Rekrutierung fakultätsinterner Dozenten themenabhängig sehr variierte ( Abb. 1). Zum Wintersemester 2007/2008 immatrikulierte sich der zweite MaReCuM-Jahrgang, sodass sich die oben aufgeführte Lehrbelastung verdoppelte.

Es soll nicht unerwähnt bleiben, dass aufgrund des externen Dozentenpools kurzfristige Dozentenausfälle kompensiert werden konnten und es zu keinem Unterrichtsausfall während dieser Zeit kam.

\section{Ůbernahme von Prüfungsaufgaben}

Eine Besonderheit von MaReCuM ist die regelmäßige Überprüfung des Lernerfolges durch Multiple-Choice(MC)-Klausuren, die alle 3 Wochen stattfinden. Hierdurch werden die Studierenden zum kontinuierlichen Lernen angehalten. Pro Jahrgang mussten so 250 MC-Fragen für das Fach Biochemie und 90 Fragen für das Fach Zellbiologie ausgesucht bzw. erstellt werden, um den Bedarf für die Klausuren und Nachklausuren zu decken. Ferner
Tab. 2 Seminarthemen im Fach Molekulare Zellbiologie.

1. Zelle und Zellkompartimentierung

2. Biomembranen

3. Zellkern und Chromosomen

4. Klassische Genetik

5. Zytoskelett

6. Zell-Matrix-Interaktionen

7. Zellzyklus, Zellteilung und Zelltod

8. Meiose

9. Endo- undExozytose, Vesikel-Transport

10. Lysosomen und Peroxisomen

11. Migration, Invasion und Metastasierung

12. Einführung in die Stammzellforschung

Tab. 3 Praktikumsblöcke in der Biochemie.

1. Grundlagen des biochemischen Experimentierens

2. Nukleinsäuren

3. Aminosäuren und Proteine

4. Enzyme und Enzymkinetik

5. Kohlenhydrate

6. Lipide

7. Blutgerinnung

8. Hormone I

9. Hormone II

10. Immunologie I

11. Immunologie II

12. Lipoproteine

13. Biochemie der Verdauung

14. Molekulargenetik I

15. Molekulargenetik II

mussten mündliche Nachprüfungen durchgeführt werden und der mündliche Teil der M1-Prüfung für das Fach Biochemie in 104 Fällen von externen Prüfern übernommen werden. Hierzu wurden aus dem externen Dozentenpool habilitierte Mitarbeiter mit langjähriger Prüfungserfahrung seitens des Dienstleisters eingesetzt.

\section{Koordinierung, Logistik und Verwaltungs- aufgaben}

Stundenplanentwicklung, Verfassen von Lehr- und Lernzielkatalogen, Teilnahme an Koordinierungssitzungen und Modulbesprechungen, Erstellen von Klausuren sind nur einige der Aufgaben, die ebenfalls vom externen Dienstleister übernommen werden können und teilweise müssen, wenn von Seiten der Hochschule noch keine Institutsstruktur verfügbar ist. Darüber hinaus kann er als Vertreter des Faches auch als direkter Ansprechpartner der ca. 360 Studierenden auftreten. Beratung oder das Erstellen von Gutachten gehören damit ebenso zu den Aufgaben wie Klausurbesprechungen.

\section{Studentische Evaluation und Ergebnisse der M1-Prüfung \\ $\nabla$}

Bei der Einrichtung des Modellstudiengangs mit dem Ausbau eines neuen Studienabschnitts wurden seitens der Fakultät systematische Evaluationsmaßnahmen zur Messung der Lehrqualität vorgenommen, deren Ziel die Verbesserung und Qualitäts- 


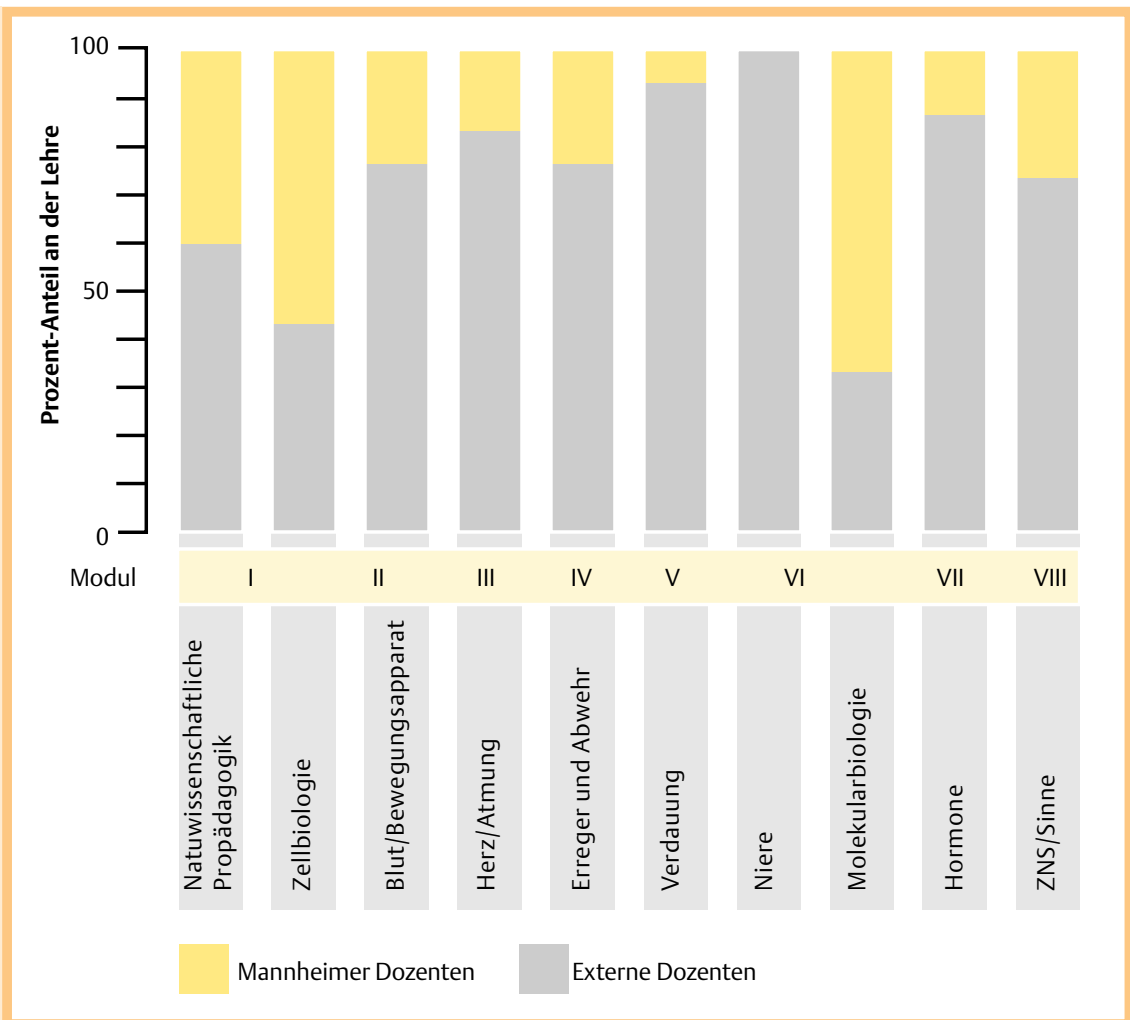

Abb. 1 Themenbezogene Verteilung fakultätseigener und externer Dozenten.

sicherung der Lehre ist. Hierzu wurde einerseits eine studentische Evaluation im Sinne einer Akzeptanzevaluation eingeführt sowie andererseits zur longitudinalen Betrachtung der Progresstest zur Erfassung des studentischen Leistungs- und Entwicklungsstands etabliert. Hierbei zeigte sich kein Unterschied zwischen Bereichen, die durch externe Dozenten vertreten wurden, gegenüber Bereichen, die durch herkömmliche Lehrstrukturen vermittelt wurden. Die Akzeptanz der Lehrveranstaltungen, die vom externen Dozentenpool gehalten wurden, war sehr hoch, so war die Anwesenheit der Studierenden bei nicht-unterschriftspflichtigen Lehrveranstaltungen über $70 \%$.

Das Humanmedizinstudium hat darüber hinaus den Vorteil, dass bundesweit nach Abschluss des Grundstudiums eine einheitliche Prüfung (M1-Prüfung; „Physikum“) die Vergleichbarkeit der Fakultäten zulässt. Dies war für den Reformstudiengang MaReCuM im Sommer 2008 das erste Mal soweit. Hierbei konnten die Mannheimer Studierenden im bundesweiten Vergleich nach den Heidelberger Studierenden den zweiten Platz belegen [5]. Dies zeigt, dass das innovative Konzept von MaReCuM sowohl inhaltlich als auch strukturell aufgegangen ist und belegt eindrucksvoll, dass Teilbereiche der universitären Lehre ohne Qualitätseinbußen an einen spezialisierten, externen Dienstleister mit einem qualifizierten Dozentenpool, der auf Honorarbasis die Sicherstellung der Lehre übernimmt, übertragen werden können.

\section{Übergang zur regulären Fachvertretung $\nabla$}

Ein weiterer Vorteil, der sich aus der externen Vertretung eines Faches ergibt, ist der reibungslose und situationsabhängig steuerbare Übergang zur regulären Fachvertretung durch eine(n) Lehrstuhlinhaber(in). Bei einer Neuberufung ergibt sich naturgemäß zunächst eine Phase, in der die Personalstruktur aufge- baut werden muss. Hier besteht die Möglichkeit, dass Personallücken im Bereich der Lehre in einer Übergangsphase noch durch den externen Dienstleister geschlossen werden. Darüber hinaus wurden alle erstellten Unterrichtsmaterialien (Seminarfolien, Praktikumsskripten, Protokolle) von der Fakultät erworben. Sie stehen also uneingeschränkt der neuen, regulären Fachvertretung zur Verfügung.

\section{Finanzieller Rahmen $\nabla$}

Ungeachtet einer Situation, in der die dringende Notwendigkeit besteht, externe Unterstützung in Anspruch zu nehmen, stellt sich die Frage, ob die Kosten eines solchen Vorgehens auch unter weniger dringlichen Umständen vertretbar sind.

Die Übernahme universitärer Lehre durch einen externen Dienstleister bedeutet, dass dieser selbstverständlich betriebswirtschaftliche Maßstäbe anlegen muss, auch in der Honorierung der Dozenten. Ferner fallen bei der Vermittlung externer Dozenten neben dem Dozentenhonorar Reisekosten an.

Um die Frage der Wirtschaftlichkeit zu beantworten, muss zunächst der Personalbedarf betrachtet werden, der notwendig gewesen wäre, um die aufgeführten Aufgaben zu übernehmen. Von 6/2006 bis 2/2009 fielen 2792 Unterrichtsstunden für externe Dozenten an und 1975 Stunden für Administrations- und Koordinierungsarbeiten (Laborausstattung, Stunden-/Dozentenplan, Lehr- und Lernzielkataloge, Modulsteckbriefe, Koordinierungssitzungen und Modulbesprechungen, Klausurerstellung, Prüfungstätigkeit, Protokollkorrekturen, Studentenberatung, Berufungskommissionssitzungen etc.). Es wurden ferner 975 Stunden Verwaltungsarbeit (Schriftwechsel, Personalverwaltung, Abrechnung, Dozentenlogistik etc.) geleistet. Für die Erstellung und Überarbeitung des Unterrichtsmaterials (Folien, Skripten, Protokollvorlagen) wurden 1150 Stunden benötigt. 
Damit ergibt sich eine Gesamtarbeitszeit von 5707 Stunden für wissenschaftliches Personal und 975 Stunden für externes Verwaltungspersonal in den bisher 33 Monaten. Aufgrund der breiten Themenpalette und der vorgegebenen Seminar- und Praktikumsstruktur wären mindestens zwei Lehrprofessuren sowie 4 wissenschaftliche Mitarbeiter mit ausschließlich Lehraufgaben hierfür notwendig. Ferner wäre eine halbe Verwaltungsstelle nötig gewesen. Kurzfristige Personalausfälle deckt dieser Personalschüssel selbstverständlich nicht ab. Bei Gegenüberstellung der Kosten dieser Aufstellung ergibt sich, dass die Kostenstrukturen in beiden Modellen ähnlich sind.

\section{Teach Pool - eine Dozentenkartei für universitäre Lehraufgaben \\ $\nabla$}

Das aufgeführte Fallbeispiel MaReCuM hat aufgezeigt, dass es möglich ist, erfolgreich universitäre Lehre von externer Seite zu vertreten. Dieser Erfolg steht und fällt in der Zukunft mit der Qualität und der Verfügbarkeit von Dozenten. Dem sich hieraus ergebenden Bedarf an Dozenten wurde durch die Entwicklung der Dozentendatei „Teach Pool“ (www.ccg-berlin.de) Rechnung getragen. In dieser Datei lassen sich die Lehrkompetenzen potenzieller Dozenten erfassen und stehen einer Vermittlung zur Verfügung. Durch Evaluierung wird die Qualität der vermittelten Dozenten erfasst und so eine qualitativ hochwertige Lehre sichergestellt.

\section{Outsourcing universitärer Lehre senkt die Lehrbelastung und schafft höhere Forschungskapazitäten \\ $\nabla$}

Der Anstieg der Lehrbelastung führt zu unterschiedlichen Lösungsansätzen. Neben der häufig üblichen, allerdings nicht produktiven Einbeziehung von Drittmittelkräften aus dem Forschungsbereich wird die Einführung von Lehrprofessuren diskutiert. So hat der Wissenschaftsrat empfohlen, Professuren mit Schwerpunkt Lehre einzuführen, die sich vorzugsweise aus den Juniorprofessuren rekrutieren sollen [6]. Ein weiterer Ansatz, wie er beispielsweise von der physikalischen Fakultät der Universität Heidelberg beschritten wurde, ist die Einführung eines Fakultätsdeputats. Hierbei ergeben sich anstelle der festen Zahl von Semesterwochenstunden flexible Lehrverpflichtungen für die verschiedenen Hochschullehrer, die sich im Laufe der Zeit ausgleichen sollen. So können Forschungsfreiräume geschaffen werden. Ob sich die Erwartungen tatsächlich erfüllen wird sich am Ende der dreijährigen Probephase zeigen [7]. Insgesamt ist allgemein erkannt worden, dass die Qualität der Lehre eine Grundvoraussetzung für die Wettbewerbsfähigkeit des deutschen Hochschulsystems ist. Der Weg dorthin wird allerdings noch kontrovers diskutiert. Der Wissenschaftsrat forderte kürzlich von den Hochschulen, dass sie in den nächsten Jahren ein umfassendes System der Qualitätsentwicklung aufbauen, um die Lehre zu verbessern. Sie sollen dabei von der von der Kultusministerkonferenz beschlossenen Systemakkreditierung unterstützt werden. Zur Finanzierung der vorgeschlagenen Maßnahmen wurden zusätzliche Mittel von jährlich 1,1 Mrd. Euro veranschlagt [8]. Die gewünschte Qualität der Lehre bedarf aber weniger eines bürokratischen Überbaus, sondern ist Resultat des Engagements der Lehrenden. Nur eine Chancengleichheit in der Karriereplanung und die gleichwertige Anerkennung von Lehr- und Forschungsleistung wird dazu führen, dass junge Akademiker diesen Weg in dem gewünschten Ausmaß einschlagen. Es bleibt allerdings festzustellen, dass wir in Deutschland diese gleichwertige Anerkennung noch nicht haben.

Das geschilderte Fallbeispiel an der Medizinischen Fakultät Mannheim hat klar belegt, dass die Verlagerung universitärer Lehraufgaben außerhalb des universitären Personalpools einen hohen Qualitätsstandard gewährleisten kann. Zudem ist dieser Weg eine angemessene Möglichkeit, um personelle Kapazitäten für Forschungsaktivitäten freizusetzen. Er dürfte eher dazu führen, die gewünschten Ziele zu erreichen, als Professuren in Forschungs- und Lehrprofessuren aufzuteilen. Lehrinhalte können durch einen externen Dozentenpool flexibler und mit einem größeren Spektrum abgedeckt werden, als dies durch einzelne Personen dauerhaft gewährleistet wäre. Darüber hinaus bliebe es dem/der Lehrstuhlinhaber(in) vorbehalten festzulegen, welche Themen gelehrt werden bzw. welche Themen institutseigenen und externen Dozenten zugeordnet werden. Dadurch könnte man auf der einen Seite aktuelle Forschungsbezüge herstellen und der eigenen Lehre ein eigenes Profil geben und auf der anderen solides Basiswissen vermitteln lassen. Durch diese Professionalisierung der Lehre wird ein höherer Grad an Einheit von Forschung und Lehre erreicht, als durch eine Trennung in Lehrund Forschungsprofessuren. Die geschaffenen Forschungsfreiräume könnten zudem dazu führen, dass forschungsaktive Hochschullehrer an den Universitäten verbleiben und nicht in reine Forschungsinstitutionen oder gar in das forschungsfreundlichere Ausland abwandern. Von ihrem Wissen können so Studierende weiter profitieren. Diese Punkte sind die stärksten Argumente für das hier vorgestellte Outsourcing von Lehraufgaben.

\section{Abstract}

\section{Outsourcing of Academic Teaching - A Perspective for the Future?}

$\nabla$

Innovative solutions are called for to deal with the increasingly heavy burdens placed on teaching staff in German universities by even higher numbers of student enrolments. Alongside differentiating between research and teaching tenures, as recommended by the German Council of Science and Humanities (Wissenschaftsrat), there is also a series of other options for meeting the challenges. One of these which has proven its value is partial outsourcing of the teaching load to external academic staff provided by a specialised service provider. Such a professionalised approach to teaching leads to a high degree of unity between research and teaching activities, whilst the wide scope for research activities allows to stop the drift of university staff engaged in research into pure institutes of research, meaning that they continue to contribute their expertise to ensure high standards in university teaching. 


\section{Literatur}

1 Haas B. Zahl der Studienanfänger auf neuem Höchststand. Berliner Morgenpost vom 2.12.2008

2 Detmer H, Lenk A. Berufungsverfahren in Deutschland. Forschung und Lehre, 10/2007

3 Schimank U, Winnes $M$. Jenseits von Humboldt? In: Stölting E, Schimank U. Die Krise der Universitäten. Wiesbaden: Westdeutscher Verlag, 2001

4 Fritz HM, Hoffmann K. Die besten Seiten von MaReCuM - Ein Leitfaden für Studierende. Mannheimer Institut für Public Health, 7/2008
5 Schriftlicher Teil des Ersten Abschnitts der Ärztlichen Prüfung. Herbst 2008. Institut für medizinische und pharmazeutische Prüfungsfragen (www.impp.de) Im Internet: www.impp.de

6 Pressemitteilung des Wissenschaftsrats vom 29.1.2007

7 van Bebber F. Einer trage des anderen Lehrbelastung. Tagesspiegel vom 28.7.2008,

8 Pressemitteilung des Wissenschaftsrats vom 7. 7.2008 